\title{
Integrated design course of applied optics focusing on operating and maintaining abilities
}

\section{Zhongjie Xu, Yu Ning, Tian Jiang, Xiangai Cheng}

Zhongjie Xu, Yu Ning, Tian Jiang, Xiangai Cheng, "Integrated design course of applied optics focusing on operating and maintaining abilities," Proc. SPIE 10452, 14th Conference on Education and Training in Optics and Photonics: ETOP 2017, 104525F (16 August 2017); doi: 10.1117/12.2269745

SDIE Event: 14th Conference on Education and Training in Optics and Photonics, ETOP 2017, 2017, Hangzhou, China 


\title{
Integrated Design Course of Applied Optics Focusing on Operating and Maintaining Abilities
}

\author{
Zhongjie $\mathrm{Xu}{ }^{1,2,3,4,5}$, Yu Ning ${ }^{1,2,3}$, Tian Jiang ${ }^{1,2,3,4}$ and Xiangai Cheng ${ }^{1,2,3,4^{*}}$ \\ ${ }^{1}$ College of Opto-Electronic Science and Engineering, National University of Defense Technology, \\ Changsha, Hunan, China \\ ${ }^{2}$ Hunan Provincial Key Laboratory of High Energy Laser Technology, Changsha, Hunan, China \\ ${ }^{3}$ Hunan Provincial Collaborative Innovation Center of High Power Fiber Laser, Changsha, Hunan, \\ China \\ ${ }^{4}$ State Key Laboratory of High Performance Computing, National University of Defense Technology, \\ Changsha, Hunan, China \\ ${ }^{5}$ Shanghai Institute of Technical Physics, Chinese Academy of Sciences, No.500 Yu Tian Road, \\ Shanghai, China
}

\begin{abstract}
The abilities of operating and maintaining optical instruments are crucial in modern society. Besides the basic knowledge in optics, our optics courses also focus on the training on handling typical optical equipment. As the link between classroom courses on applied optics and the field trips, the integrated design course of applied optics aims to give the students a better understanding on several instantly used optical equipment, such as hand-held telescope and periscope, etc. The basic concepts of optical system design are also emphasized as well. The course is arranged rightly after the classroom course of applied optics and composed of experimental and design tasks. The experimental tasks include the measurements of aberrations and major parameters of a primitive telescope, while in the design parts, the students are asked to design a Keplerian telescope. The whole course gives a deepened understandings on the concepts, assembling, and operating of telescopes. The students are also encouraged to extend their interests on other typical optical instruments.
\end{abstract}

Keywords: Integrated Design, Applied Optics, Optical Instruments.

\section{INTRODUCTION}

After the invention of astronomical telescope by Galileo Galilei and microscope by Antony van Leeuwenhoek, optical instruments had become powerful and essential tools in scientific research, industry, social lives and military. The science and technologies on optical instruments have been well developed within the past several hundred years. Nowadays, the abilities of designing, operating and maintaining optical instruments are crucial in related aspects. The education systems on this field are well established around the world, and a number of outstanding text books in multiple languages are available[1-3]. In China, the courses related on the basic knowledge of optical instruments are usually called applied optics. The course series is usually consist of fundamental concepts, design skill practices and operating trainings. A number of universities have paid more attentions on the improvements of design skills in order to meet the requirements of scientific researches and industry. However, in some fields, the abilities of operating and maintaining typical optical instruments are of much more importance.

Besides the basic knowledge of optical instruments, the course series of applied optics in the National University of Defense Technology is mainly aimed for improving the abilities of operating and maintaining typical optical instruments of undergraduates. The integrated design course of applied optics is one of the important parts of the whole series. It is the link between classroom studies and field trip courses. The purposes of this course are giving the students a better understanding on several instantly used optical equipment, as well as the emphasizing of important concepts. The whole

*E-mail: xiang_ai_cheng@126.com

14th Conference on Education and Training in Optics and Photonics: ETOP 2017, edited by Xu Liu,

Xi-Cheng Zhang, Proc. of SPIE Vol. 10452, 104525F - ㅇ 2017 ICO, IEEE, OSA, SPIE

CCC code: $0277-786 \mathrm{X} / 17 / \$ 18 \cdot$ doi: $10.1117 / 12.2269745$ 
course contains both experimental and design parts, which will be described in detail below. This article is prepared as the following structure: in section 2 a brief introduction about the relative courses is presented. In section 3 and 4 we will introduce the main tasks in experimental and design parts, respectively. A brief conclusion is given in section 5.

\section{RELATED COURSES}

The course series is mainly for undergraduate students. The basic course is optics, which gives basic description of wave optics and ray optics. The concepts of paraxial approximation, interference and diffraction are introduced in this course. Generally, the content of this course has no much differences with that in other universities.

After the students have finished the course of optics, they are asked to attend the course of applied optics, which is the major course of this series. The concepts in coaxial spherical systems are emphasized in order to give a fundamental picture about surfaces, lens, and optical systems. The human eyes, telescopes and microscopes are discussed as the examples of coaxial spherical systems. After that, prisms are introduced, and the periscope is discussed as an example. The theory of aberration is briefly talked as the last component of basic knowledge part, while the colorimetry and photometry are introduced in individual graduate courses. After the basic knowledge part is finishing, the students are asked to organize several theme talks including astronomical telescope, microscope, 3D camera, projector, optical fiber and infrared systems. During the preparation of these theme talks, the students will learn various optical instruments. With the help of the basic knowledge, the students will get a basic idea on designing, operation and maintaining optical instruments.

The integrated design course of applied optics is rightly after the course of applied optics. The course lasts for 2 weeks (ten days). The whole time is divided into two parts as follows: In the first day, we review the geometry optics and basic aberration theory. In the last 9 days, the students are divided into two groups to finishing experimental and design tasks alternatively. The students will get deepened understanding about the structure of typical optical instruments.

After that, the students are organized to have a field trip, in which they will get in touch with several typical optical equipment, and learn to operate and assemble the equipment.

There are also some related courses for graduate students. One as an example is the fiber optics. The students will the concepts and measurements about the optical fibers, especially their usage in communication and laser.

\section{EXPERIMENTAL TASKS}

The experiment part contains three tasks: the observing and measuring of monochromatic aberrations, the observing of simple periscopes and the assembling and measuring of telescopes, which refer to basic theory, structure of optical instruments and assembling of optical instruments, respectively.

\subsection{The observing and measuring of monochromatic aberrations}

The task contains the following contents:

(1) Observe monochromatic aberrations.

The students are allowed to use following elements: one collimator with a point source or cross-shaped source on its focus, several sample lens, one CCD camera and other mechanical elements. The sample lens are designed to have particularly large spherical, comatic, astigmatic aberrations and field curve, respectively. The students are asked to observe and record the typical spots of monochromatic aberrations (include spherical, comatic and astigmatic aberrations) near lens' focus points. They are also encouraged to find out how to observe field curve. The main aim of this experiment is let students get a direct impression of different aberrations. When they encounter similar aberrations in the future, they are expected to recognize the type of aberrations and find out way to correct them.

An example of the student work is in Fig.1, which are identical with typical results. The field curve is observed though the different clarities between center and side lines in Fig. 1(c). 

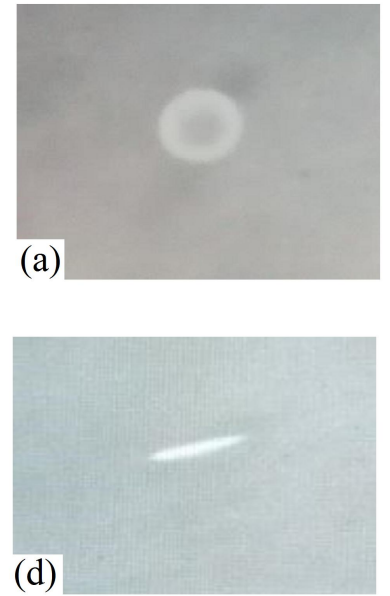
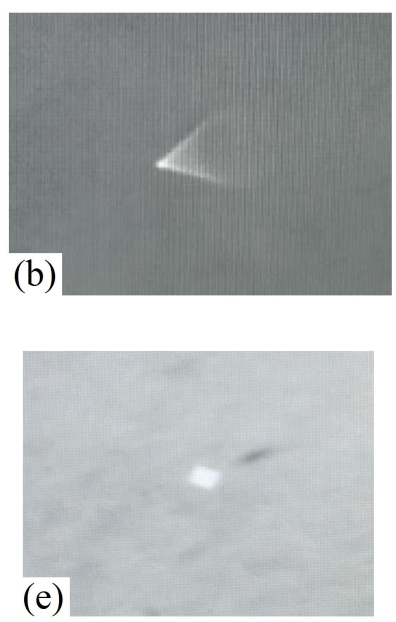
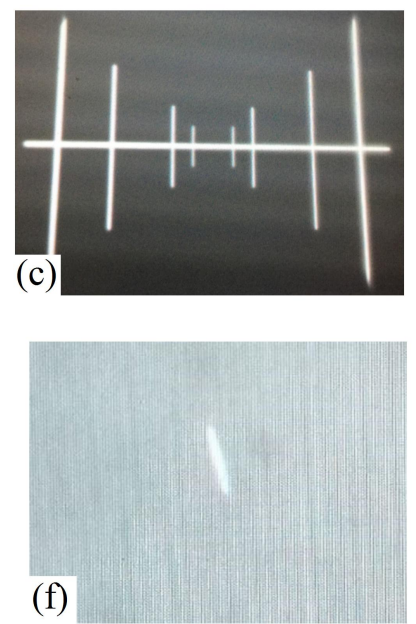

Figure 1 Example of monochromatic aberrations. (a) Spherical aberration. (b) Coma. (c) Field curve. (d)-(f) Astigmatism before, at and after focus, respectively.

(2) Observe and measure aberrations with shadow method.

The students are allowed to use following elements: One parallel light source, lens with large spherical aberrations and coma, respectively, graduated screen, and a stop with tunable blade. The stop is placed near the back focus of lens. The students are asked to observe the spot shapes after the stop, and measure the influences of stop positions on the spot shapes. This experiment is a practice on the measuring of aberrations.

An example of the student work is in Fig.2. Fig.2(a) is the spot shapes for spherical aberration lens while the stop is at different positions, and Fig.2(b) is the corresponding spherical aberration curve. Fig.2(c) is the spot shapes for coma lens.

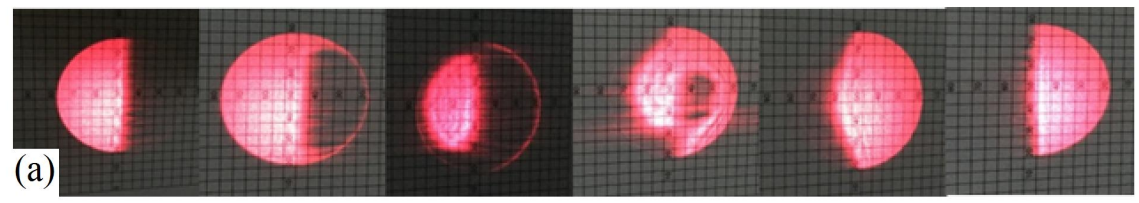

(b)

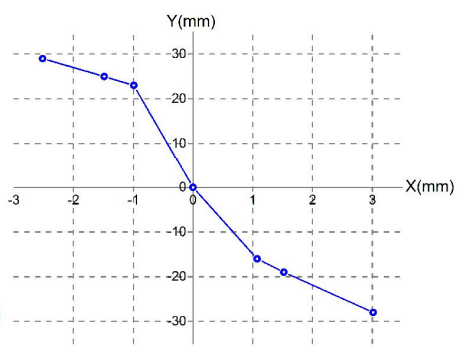

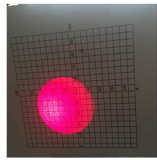

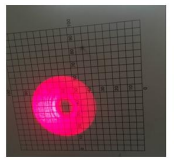

(c)
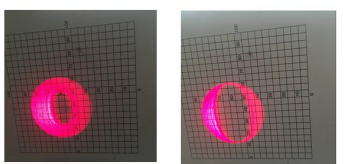

Figure 2 Example of spherical aberrations and coma. (a) Spot shapes for spherical aberration. (b) Spherical aberration curve. (c) Spot shapes for coma.

\subsection{The observing of simple periscopes}

A simple periscope with its light path shown in Fig.3 is presented to students. The students are asked to analyze the contribution of every lens and prism and figure out the chirality of images after each element. The students are encouraged to make some measurements of distant objects with this periscope. 


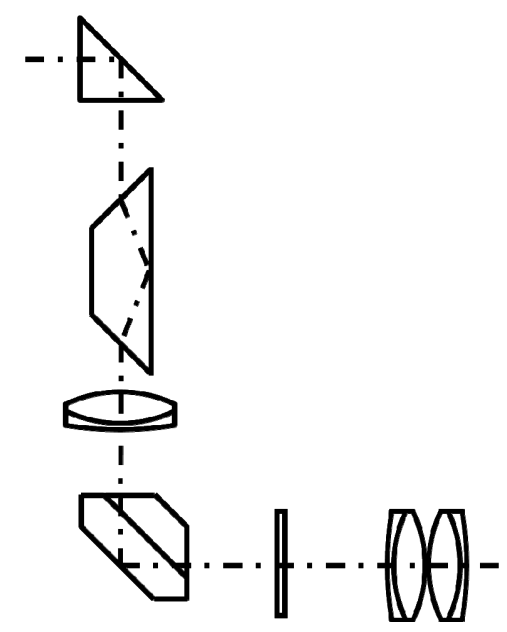

Figure 3 The light path of simple periscope.

This is a task without much handwork. The main aim is to make students get familiar with typical periscopes and have better understanding on the effects of prisms and the chirality of images.

\subsection{The assembling and measuring of telescopes}

The students are provided with the following elements: one collimator with multiple line source on its focus plane (The line space of source and the focal length is known), one objective and eyepiece with unknown focal length, one objective with known focal length, one graduated eyepiece with known $\gamma$, one screen, one stop and other mechanical elements. The students are asked to properly assemble a telescope with objective and eyepiece with unknown focal length, and then measure the angular magnification of the telescope.

The main aim of this experiment is let students know how to assemble simple telescopes, how to measure the telescopes, and the effect of stop.

Two different setups are found in student work, whose light paths are shown in Fig.4. In both setups OBJ 2 is placed right in the exit pupil of the assembled telescope. At Fig.4 (a), the image through OBJ 2 is observed and measure with a graduated eyepiece, while in Fig.4 (b), the image is caught by a screen and the image size is measured by ruler. Both setups can reach correct answer of this task with slightly different calculating procedures. A statistics of the measured value of angular magnification by different groups are shown in Fig.5. The blue dashed line indicates the exact value. Most groups with correct setups have get close to the exact value. The deviations may be caused by imprecise positions of lens focus.

(a)
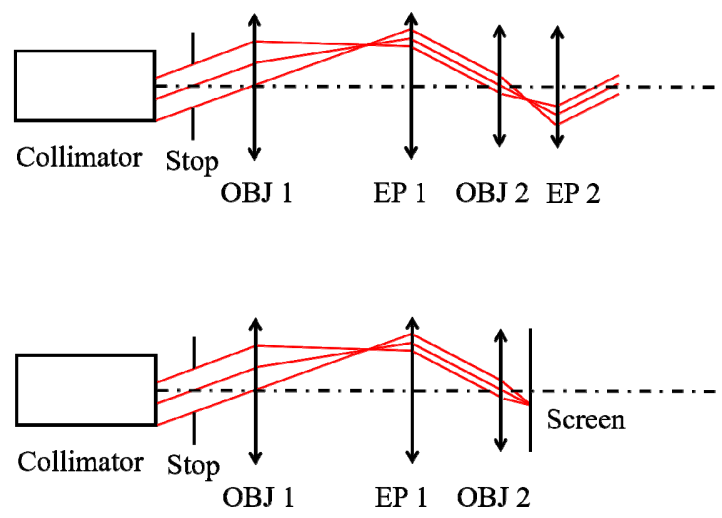

(b)

Figure 4 Light paths of task 3. The focal length of OBJ 1 and EP 1 is not provided. (a) The grades on EP 2 are read by eyes. (b) The image is in the screen and its size is measured. 


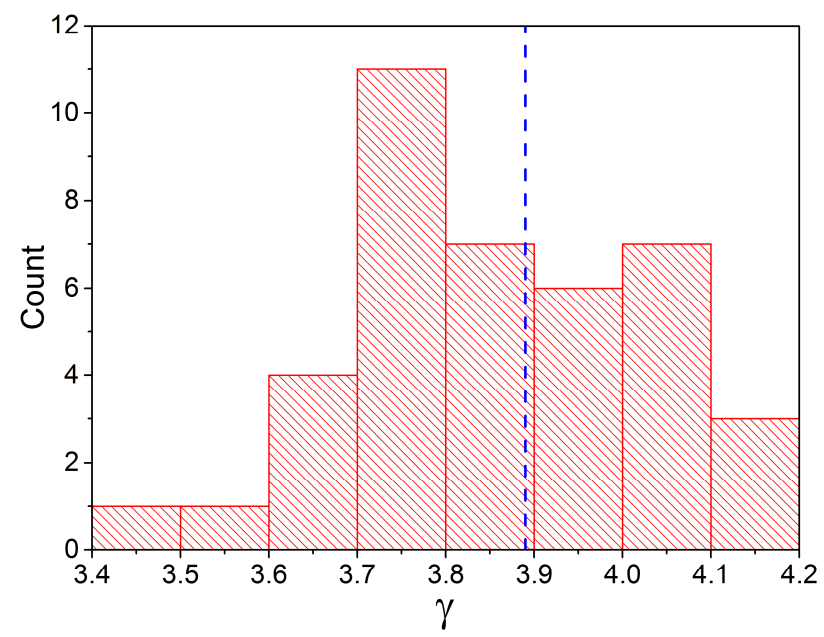

Figure $5 \mathrm{~A}$ statistics of the calculated value of angular magnification by different groups.

\section{DESIGN TASKS}

The design tasks are focused on the telescopes. The students are asked to design optical systems for an 8X Keplerian telescope with Zemax. The detailed requirements are as follows:

Design Task: Optical System of Keplerian Telescope

(1) The angular magnification is fixed to $8 \mathrm{X}$.

(2) The focal length of eyepiece is $25 \mathrm{~mm}$.

(3) The exit pupil is $4 \mathrm{~mm}$.

(4) The field angle of eyepiece is $2 \omega^{\prime}=25^{\circ}$.

(5) The distance of exit pupil is larger than $22 \mathrm{~mm}$.

(6) The telescope is designed for visible light.

This design task is fairly simple. It is worth to mention that the task is not aiming for the training of "design skills", but rather the deepened understandings on assembling typical optical instruments and the concepts of aberration and MTF.

An example of the student work is in Fig.6. The objective is consist with a doublet and two singlet, and the eyepiece is of Alf type. A paraxial lens with a focal length of $16.67 \mathrm{~mm}$ is placed in the exit pupil to analyze the MTF. Since the telescope uses rather large number of lens, the MTF is fairly good, which is shown in Fig.6 (b). 


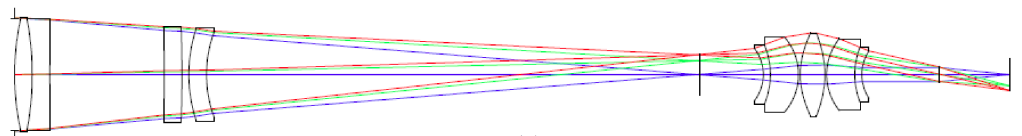

(a)

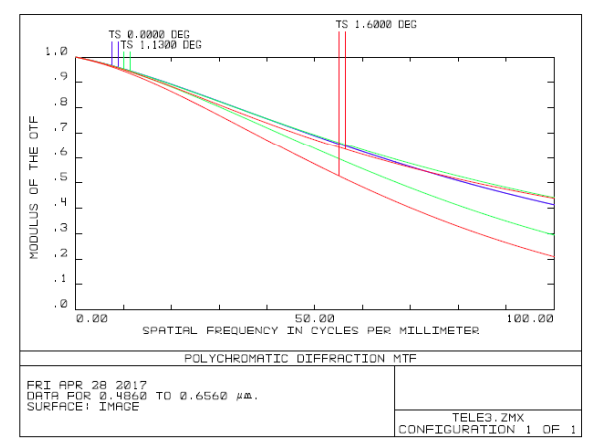

(b)

Figure 6 An example of the student work. (a) The light path. (b) The MTF.

\section{CONCLUSION}

The integrated design course of applied optics in national university of defense technology is aimed at training the abilities of assembling, operating and maintaining typical optical instruments. The whole course is divided into experimental and design parts. Both parts gives students deepened understandings on the concepts and improving their operating abilities. In the past 4 years, the course has made significant positive effects on undergraduate students.

\section{REFERENCES}

[1] Li, L. Applied Optics, Beijing Institute of Technology Press, 2006.

[2] Fischer, R. E., Optical System Design, McGraw-Hill, 2000.

[3] Smith, W. J. Modern Optical Engineering, McGraw-Hill, 2000. 